\title{
Prevalence of Breast Cancer and Its Associated Factors for Recurrence in Hospital Tengku Ampuan Afzan 2008 - 2012
}

\author{
Azhani Chik \\ International Islamic University Malaysia
}

Introduction: Breast cancer is the commonest malignancy in Malaysian women. Cancer recurrence has been a detrimental factor towards survival with peak of recurrence recorded in first 2 years of diagnoses. Identifying the prognostic factors towards recurrence is important to management and prolonging survival. Materials and method: We have retrospectively analyzed 179 patients women with breast cancer based on 5 years single centre database with minimum follow up of 2 years. The demographic and clinicopathological characteristics were determined using descriptive statistics. Survival were calculated based on Kaplan- Meier method and multivariate analysis by Cox proportional hazards was performed to evaluate the potential factors affecting breast cancer recurrence. Results: Mean follow up was 42 months, with mean age 52 years and $60.9 \%$ presented in Stage II disease. Overall recurrence was $41.9 \%$ with local recurrence $2.1 \%$, regional recurrence $12.3 \%$ and distant recurrence $27.4 \%$. 50\% of our patients developed recurrence at 25 months. In univariate analysis, time to first presentation was significantly correlated with recurrence. However, in multivariate analysis; tumor size, lymph node positivity and lymphovascular invasion were independently associated with recurrence. Conclusion: Even though local data on breast cancer recurrence is sparse, it does correlate with the international data. Thus, optimizing our care in breast cancer. 